\title{
Síndrome Meckel-Gruber: diagnóstico prenatal. Reporte de un caso en el sureste de México
}

\author{
Meckel-Gruber syndrome: prenatal diagnosis. Report of a case in Southeast Mexico \\ Daniela del Carmen Aquino Bonilla, * Ever Domínguez Morales, ${ }^{\ddagger}$ \\ Leonardo Ulises García Pérez, $\$$ Clara Magdalena Martínez Hernández
}

Citar como: Aquino BDC, Domínguez ME, García PLU, Martínez HCM. Síndrome Meckel-Gruber: diagnóstico prenatal. Reporte de un caso en el sureste de México. Acta Med. 2020; 18 (4): 418-421. https://dx.doi.org/10.35366/97271

\section{Resumen}

Introducción: El síndrome Meckel-Gruber es una enfermedad autosómica recesiva causada por disfunción ciliar primaria durante la embriogénesis temprana. Su incidencia es variable y depende de la región geográfica y el origen étnico. Caso clínico: Femenino de 21 años de edad, referida por centro de segundo nivel debido a embarazo de 19 semanas de gestación y feto con malformaciones. Se realiza ecografía estructural que reporta encefalocele occipital y displasia quística renal bilateral, se integra el diagnóstico ecográfico de Meckel-Gruber. Con base en lo anterior (pronóstico malo para función y vida del feto) se propone a la pareja interrupción del embarazo. Se efectúa estudio anatomopatológico que describe encefalocele occipital, displasia quística renal y polidactilia postaxial izquierda. Con base en los hallazgos ecográficos y anatomopatológicos se integra el diagnóstico definitivo de Meckel-Gruber. Conclusión: El síndrome Meckel-Gruber es una patología que presenta un pronóstico letal para la vida y función del feto. Es importante realizar en forma temprana el diagnóstico prenatal del padecimiento, ello permitirá establecer un adecuado manejo genético-reproductivo posterior.

Palabras clave: Meckel-Gruber, encefalocele occipital, diagnóstico prenatal.

* Médico residente de cuarto año en Ginecología y Obstetricia.

* Médico adscrito al Departamento de Genética.

$\S$ Jefe del Servicio de Biología de la Reproducción.

"Jefa de Investigación.

Hospital Regional de Alta Especialidad de la Mujer (HRAEM), Villahermosa, Tabasco, México.

Correspondencia:

Dr. Ever Domínguez Morales.

Correo electrónico: everdguez@msn.com

Aceptado: 01-08-2020.

www.medigraphic.com/actamedica

\section{Abstract}

Introduction: Meckel-Gruber syndrome is an autosomal recessive disease; caused by primary ciliary dysfunction during early embryogenesis. Its incidence is variable and depends on the geographical region and ethnic origin. Clinical case: 21-year-old female, referred by second level center; due to a 19week pregnancy and malformed fetus. A structural ultrasound was performed that reported occipital encephalocele, bilateral renal cystic dysplasia; integrating the ultrasound diagnosis of Meckel-Gruber. Based on the above (predict poor function and life of the fetus) it was proposed to terminate the pregnancy. An anatomopathological study was carried out that described occipital encephalocele, renal cystic dysplasia, and left postaxial polydactyly. Based on the ultrasound and pathological findings, the definitive diagnosis of Meckel-Gruber is integrated. Conclusion: Meckel-Gruber syndrome is a pathology that presents a lethal prognosis both for the life and function of the fetus. It is important to carry out the prenatal diagnosis of the disease early, to allow the establishment of adequate subsequent genetic-reproductive management.

Keywords: Meckel-Gruber, occipital encephalocele, prenatal diagnosis.

\section{INTRODUCCIÓN}

El síndrome Meckel-Gruber (MKS) (disencefalia esplacnoquística, OMIM \#249000) es una enfermedad autosómica recesiva causada por disfunción ciliar primaria durante la embriogénesis temprana. Es caracterizado por la tríada siguiente: displasia quística renal bilateral (100\%), encefalocele occipital $(90 \%)$ y polidactilia postaxial $(83.3 \%) .^{1-3}$ La prevalencia estimada a nivel mundial oscila entre uno en 1,300 y uno en 140,000. Su incidencia es variable, depende de la región geográfica y del origen étnico. ${ }^{4,5}$ 
El MKS es una alteración genéticamente heterogénea, con al menos 13 diferentes genes conocidos. Los genes con mayor repercusión son: MKS1, MKS3, MKS4 y MKS6. ${ }^{6}$

El objetivo es reportar un caso de síndrome MeckelGruber diagnosticado prenatalmente, el cual presentaba los hallazgos ecográficos clásicos.

\section{CASO CLÍ́NICO}

Femenino de 21 años, primigesta, acude por referencia de centro de segundo nivel debido a embarazo de 19.4 semanas de gestación por ultrasonido del segundo trimestre, el cual reporta feto con alteraciones estructurales. Sin antecedentes heredofamiliares de importancia, antecedentes de patología genética-reproductiva negados y antecedente de consanguinidad negativa (Figura 1).

Se realizó estudio ecográfico de alta definición (estructural), el cual reportó lo siguiente: feto de 19 semanas, por fetometría promedio (tres semanas menor), se observó microcráneo a expensas de encefalocele occipital con gran cantidad de materia protruyendo la cavidad amniótica, derrame pericárdico de predominio derecho y probable transposición de grandes vasos. El tamaño abdominal fue excesivo a expensas de nefromegalia bilateral, los pies con talones prominentes; no se observó distensión de cámara gástrica ni vejiga. Debido a posición fetal y líquido amniótico subjetivamente disminuido no fue posible confirmar la cuenta de dedos de manos y pies. La impresión de diagnóstico desde el punto de vista ecográfico fue síndrome Meckel-Gruber (debido al encefalocele occipital y nefromegalia).

Se llevó a cabo el asesoramiento genético a los padres, explicando de forma detallada y clara los hallazgos observa-

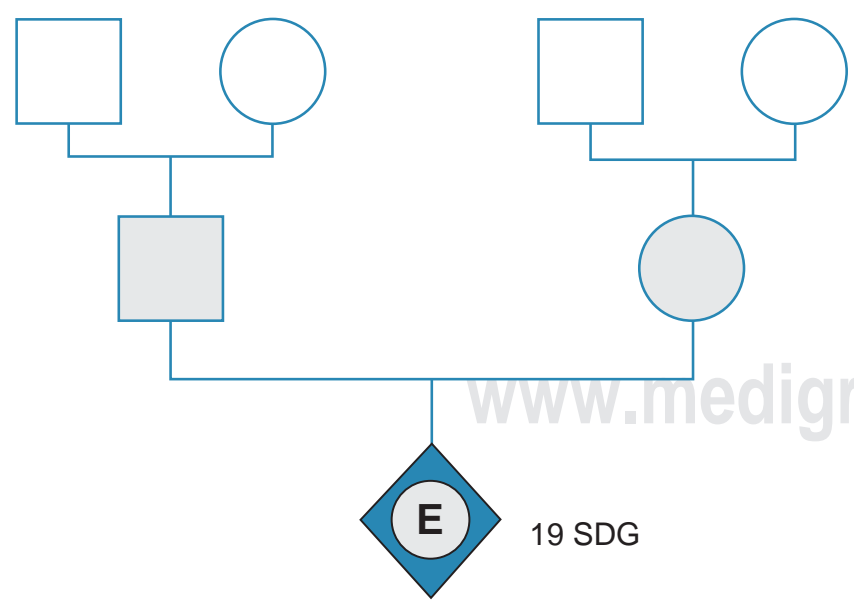

Figura 1: Árbol genealógico. SDG = semanas de gestación . dos en la ecografía, haciendo mención de que el síndrome tiene mal pronóstico para la vida y función del paciente (feto), por lo cual solicitan la interrupción del embarazo mediante consentimiento informado.

Los familiares autorizaron la autopsia, la cual fue realizada en nuestra unidad hospitalaria. El reporte del estudio anatomopatológico mencionó lo siguiente: feto masculino de 20 semanas aproximadamente con alteraciones de sistema nervioso central (encefalocele occipital) y alteraciones en la migración neural, displasia renal quística bilateral, sin anomalías obstructivas en vías urinarias, alteraciones hepáticas con proliferación de la placa limitante (fibrosis hepática congénita), alteraciones ductales en páncreas, polidactilia postaxial izquierda (Figura 2) que constituyen Meckel-Gruber.

De acuerdo con las características ecográficas y anatomopatológicas previamente descritas se estableció el diagnóstico definitivo de síndrome Meckel-Gruber.

\section{DISCUSIÓN}

El MKS fue descrito por primera vez por Johann Friedrich Meckel en 1822 en dos hermanos que murieron de malformaciones idénticas de encefalocele occipital, riñones poliquísticos y polidactilia. George B. Gruber en 1934 informó casos familiares con características similares y acuñó el término disencefalia esplacnoquística. ${ }^{7,8}$

El diagnóstico prenatal de MKS puede realizarse mediante ecografía del primero (11-14 SDG) o segundo trimestre (18-23 SDG). Ello obedece a que, dependiendo de las semanas de gestación, podrán ser detectables cada uno de los hallazgos correspondientes a la patología. 9,10 La edad gestacional en la cual se llevó a cabo el diagnóstico en nuestro caso fue de 19 semanas, similar a lo reportado en los artículos publicados.

Los criterios diagnóstico de MKS incluyen la presencia de al menos dos de los siguientes: displasia quística renal (100\%), encefalocele occipital $(90 \%)$ y polidactilia postaxial (83.3\%). En la mayoría de los casos el encefalocele es de localización occipital (75-80\%), parietal (15-20\%) y frontal (5\%). ${ }^{11,12}$ En nuestro reporte se observó que los criterios de diagnóstico y la localización del encefalocele corresponden con lo previamente informado en la literatura.

Los hallazgos anatomopatológicos que se han descrito en la literatura son: displasia quística renal, encefalocele occipital, polidactilia postaxial y displasia hepática (fibrosis, infiltración linfocitaria, alteración ductal). Es importante realizar el estudio anatomopatológico correspondiente, ello permitirá corroborar y relacionar los hallazgos ecográficos, anatomopatológicos y genéticos permitiendo un diagnóstico de certeza. ${ }^{13,14}$ En nuestro caso se realizó el estudio 

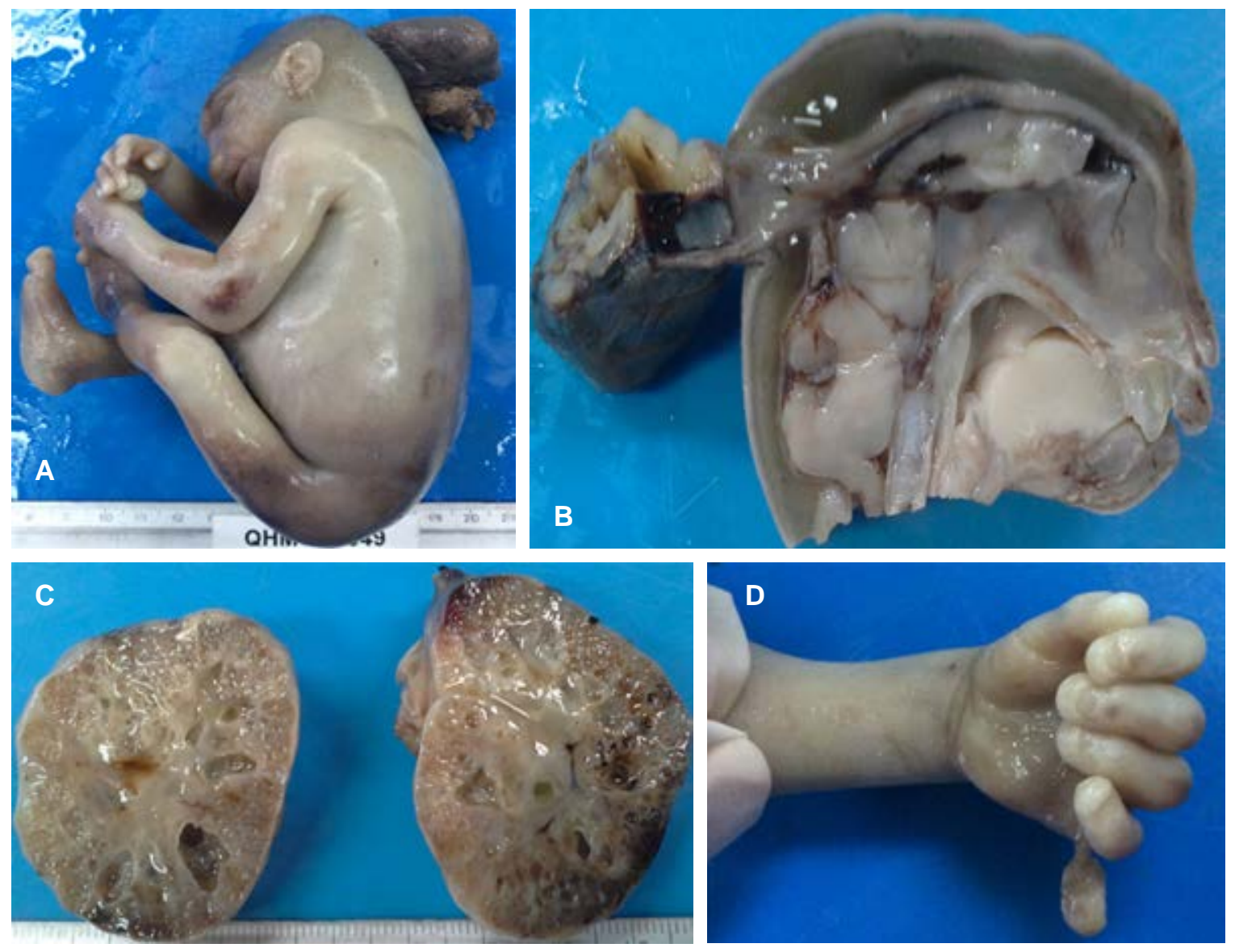

Figura 2: A) Feto con encefalocele occipital y talones prominentes. B) Encefalocele occipital (corte sagital). C) Displasia quística renal bilateral. D) Polidactilia postaxial izquierda.

Tabla 1: Diagnóstico diferencial.

\begin{tabular}{lcccccc}
\hline Síndrome & $\begin{array}{c}\text { Encefalocele } \\
\text { occipital }\end{array}$ & $\begin{array}{c}\text { Displasia } \\
\text { quística renal }\end{array}$ & $\begin{array}{c}\text { Polidactilia } \\
\text { postaxial }\end{array}$ & Herencia & Cromosoma y/o gen & Locus \\
Meckel-Gruber & Sí & Sí & Sí & Autosómica recesiva & MKS1 & $17 q 22$ \\
Trisomía 13 & No & Sí & Sí & Cromosómica & Cromosoma 13 \\
Riñón poliquístico & No & Sí & No & Autosómica recesiva & PKHD1 & $6 p 12.3-p 12.2$ \\
Smith-Lemli-Opitz & No & Sí & Sí & Autosómica recesiva & DHCR7 & $11 q 13.4$ \\
Bardet-Biedl & No & Sí & Sí & Autosómica recesiva & BBS1 & $11 q 13.2$
\end{tabular}

anatomopatológico respectivo, en el cual se observaron los hallazgos característicos descritos en la literatura.

El diagnóstico diferencial del MKS se establece con trisomía 13, riñón poliquístico autosómico recesivo, Smith-Lemli-Opitz y Bardet-Biedl, debido a que comparte datos clínicos con algunas de estas patologías. ${ }^{15}$ En nuestro caso, la presencia de los hallazgos clásicos de MKS permitieron el diagnóstico del padecimiento (Tabla 1).

El asesoramiento genético es un proceso importante que permite establecer el diagnóstico, pronóstico y manejo prenatal, el cual se llevó a cabo en nuestro caso. Para la posterior planificación genética-reproductiva de un embarazo se deberá ofrecer a las pacientes con mayor riesgo 
de recurrencia, el acceso a las pruebas de diagnóstico prenatal de acuerdo a la edad gestacional respectiva, un control prenatal estricto y las valoraciones multidisciplinarias correspondientes.

\section{CONCLUSIÓN}

El síndrome Meckel-Gruber es una patología que presenta un pronóstico letal para la vida y función del feto. Es importante realizar en forma temprana el diagnóstico prenatal del padecimiento, pues ello permitirá establecer un adecuado manejo genético-reproductivo posterior.

\section{REFERENCIAS}

1. Meckel syndrome, type 1; MKS1. (Internet). OMIM: Johns Hopkins University. [Cited 24 April 2020]. Available in: http://omim.org/ entry/249000.

2. Martínez MJ, Sanz AO, Amat VI, Azcona RB, Cabistany EA, Martín ME. Síndrome de Meckel. Diagnóstico prenatal y diagnóstico diferencial. Prog Obstet Ginecol. 2012; 55 (6): 269-273. doi: 10.1016/j. pog.2012.02.005.

3. Audifred-Salomón J, Barrita-Domínguez IJ, Ortiz de Zarate-Alarcón G, Sánchez-Hernández H, Camacho-Cervantes A. Diagnóstico prenatal de síndrome de Meckel-Gruber. Reporte de un caso y revisión de la bibliografía. Ginecol Obstet Mex. 2016; 84 (2): 105-111.

4. Ramírez-Izcoa AE, Sánchez-Sierra LE, Alvarenga-Calidonio RH, Varela-González D. Síndrome de Meckel-Gruber en un lactante menor con sobrevida prolongada. Reporte de caso. Acta Pediatr Mex. 2018; 39 (1): 33-41. doi: 10.18233/APM39No1pp33-411538

5. Ridnõi K, Šois M, Vaidla E, Pajusalu S, Kelder L, Reimand T et al. A prenatally diagnosed case of Meckel-Gruber syndrome with novel compound heterozygous pathogenic variants in the TXNDC15 gene. Mol Genet Genomic Med. 2019; 7 (5): e614. doi: 10.1002/ mgg3.614.

6. Hartill V, Szymanska K, Sharif SM, Wheway G, Johnson CA. MeckelGruber syndrome: an update on diagnosis, clinical management, and research advances. Front Pediatr. 2017; 5: 244. doi: 10.3389/ fped.2017.00244.

7. Chiriac DV, Hogea LM, Bredicean AC, Rednic R, Nussbaum LA, Hogea GB et al. A rare case of Meckel-Gruber syndrome. Rom J Morphol Embryol. 2017; 58 (3): 1023-1027. PMID: 29250684.

8. Yaqoubi HNA, Fatema N. Meckel Gruber syndrome associated with anencephaly-an unusual reported case. Oxf Med Case Reports. 2018; 2018 (2): omx092. doi: 10.1093/omcr/omx092.

9. Alam A, Adhi M, Bano R, Zubair A, Mushtaq A. Meckel Gruber syndrome: second trimester diagnosis of a case in a nonconsanguineous marriage. Pak J Med Sci. 2013; 29 (1): 234-236. doi: 10.12669/pjms.291.2930.

10. Jones D, Fiozzo F, Waters B, McKnight D, Brown S. First-trimester diagnosis of Meckel-Gruber syndrome by fetal ultrasound with molecular identification of CC2D2A mutations by next-generation sequencing. Ultrasound Obstet Gynecol. 2014; 44 (6): 719-721. doi: 10.1002/uog.13381.

11. Barisic I, Boban L, Loane M, Garne E, Wellesley D, Calzolari E et al. Meckel-Gruber syndrome: a population-based study on prevalence, prenatal diagnosis, clinical features, and survival in Europe. Eur J Hum Genet. 2015; 23 (6): 746-752. doi: 10.1038/ejhg.2014.174.

12. Fetal Abnormalities. Brain: Encephalocele (Internet). The Fetal Medicine Foundation. [Cited 05 July 2020]. Available in: https:// fetalmedicine.org/education/fetal-abnormalities/brain/encephalocele.

13. Kar A, Dhal I, Madurwar N, Kanungo S. Meckel-Gruber syndrome: autopsy based approach to diagnosis. J Forensic Sci Med. 2016; 2: 53-56. doi: 10.4103/2349-5014.165708.

14. Raj M, Dhanuka S, Agarwal P, Reddy SL, Vivekananthan S. Meckel Gruber syndrome-a case report. Surg Exp Pathol. 2020; 3: 11. doi: org/10.1186/s42047-020-00062-3.

15. Myageri A, Grampurohit V, Rao R. Meckel Gruber syndrome: report of two cases with review of literature. J Family Med Prim Care. 2013; 2 (1): 106-108. doi: 10.4103/2249-4863.109971.

Conflicto de intereses y financiamiento: Daniela del Carmen Aquino, Ever Domínguez, Leonardo Ulises García y Clara Magdalena Martínez declaran que no tienen nungún conflicto de intereses y no haber recibido patrocinio para la realización del trabajo. 